\title{
The effect of non-invasive positive pressure ventilation (NIPPV) on cognitive function in amyotrophic lateral sclerosis (ALS): a prospective study
}

\author{
I C Newsom-Davis, R A Lyall, P N Leigh, J Moxham, L H Goldstein
}

\begin{abstract}
Objectives-Neuropsychological investigations have shown a degree of cognitive dysfunction in a proportion of nondemented patients with ALS. Respiratory muscle weakness in ALS can lead to nocturnal hypoventilation, resulting in sleep disturbance and daytime somnolence. Sleep deprivation of this type may cause impairments in cognitive function, but this has not been formally evaluated in ALS.
\end{abstract}

Methods-Cognitive functioning was evaluated in nine patients with ALS with sleep disturbance caused by nocturnal hypoventilation (NIPPV group), and in a comparison group of 10 similar patients without ventilation problems (control group). The NIPPV group then started non-invasive positive pressure ventilation (NIPPV) at night. After about 6 weeks, change in cognitive function was evaluated.

Results-Statistically significant improvement in scores on two of the seven cognitive tests was demonstrated in the NIPPV group postventilation, and a trend

Department of Psychology, Institute of Psychiatry, De Crespigny Park, London SE5 8AF, UK

I C Newsom-Davis

L H Goldstein

Department of Respiratory Medicine, Guy's, King's, and St Thomas' School of Medicine, King's College Hospital, London, SE5 9PJ, UK R A Lyall

J Moxham

Department of Neurology Guy's, King's, and St Thomas' School of Medicine and Institute of Psychiatry, London SE5 8AF, UK

P N Leigh

Correspondence to: Dr L Goldstein

1.goldstein@iop.kcl.ac.uk

Received 19 October 2000 and in revised form

3 April 2001

Accepted 14 May 2001 memory..$^{5-8}$ Functional neuroimaging studies in ALS have demonstrated an association between poor executive task performance and reduced regional blood flow in the prefrontal area, ${ }^{12}$ and structural imaging has shown progressive atrophy in the frontal and anterior temporal lobes, as well as the motor cortex. ${ }^{9}$ The mechanism underlying these changes remains obscure, although it has been assumed to be closely related to the processes responsible for the neurodegenerative process. An additional - or even alternative-explanation for cognitive dysfunction in ALS is respiratory muscle weakness with impaired nocturnal ventilation and sleep disturbance.

The presence of sleep disordered breathing is well established in ALS. ${ }^{10-12}$ Weakness of the respiratory muscles, particularly the diaphragm, is invariably present as patients reach significant levels of disability ${ }^{13}$ and ventilatory failure is the most common cause of death. Earlier, milder degrees of respiratory muscle weakness may not be sufficient to compromise ventilation during the day. However, respiratory function worsens during REM (rapid eye movement) or deep sleep, as a result of the combined effects of hypotonia of the accessory muscles of respiration, a weak diaphragm, and supine position, and this can lead to nocturnal hypoventilation. During hypoventilation levels of carbon dioxide and bicarbonate in the blood rise, and arterial blood oxygen saturation falls. When oxygen saturations fall below a critical level an arousal occurs, in which the subject moves from deep to lighter sleep, the accessory muscles become active, and adequate ventilation is restored. These arousals can occur very often. Sleep disruption may be further exacerbated by muscle cramps, reduced mobility, and swallowing problems. ${ }^{14}$ Such sleep disturbance may occur in as many as $44 \%$ of patients with ALS with bulbar involvement ${ }^{15}$ and results in a range of symptoms including daytime somnolence, headaches, and loss of appetite. It is known that obstructive sleep apnoea, which causes similar symptoms to those described above, is associated with cognitive dysfunction affecting executive functions, attention, and memory. ${ }^{16-18}$ It has also been shown that successful treatment can reverse these effects. ${ }^{120}$ However, these issues have not been addressed before in an ALS population with hypoventilation.

Non-invasive positive pressure ventilation (NIPPV) has been used to treat sleep related 
respiratory problems in patients with neuromuscular disease for more than 10 years. Noninvasive ventilation can be used for intermittent, nocturnal support ${ }^{21}$ and its use has been shown to reduce morning headache in patients with ALS. ${ }^{22}{ }^{23}$ There is also recent evidence to show increased survival in patients with ALS using NIPPV, ${ }^{24}{ }^{25}$ but its impact on cognitive performance has not previously been investigated.

\section{Methodology}

PATIENTS

The NIPPV group consisted of nine patients with a diagnosis of possible, probable, or definite ALS, as defined by the El Escorial criteria, ${ }^{26}$ and evidence of hypoventilation and sleep disturbance. All had reduced respiratory muscle strength $(<80 \%$ predicted vital capacity), and nocturnal polysomnography showing episodes of nocturnal hypoventilation causing arousals. In addition, there was evidence of abnormal daytime blood gases $\left(\mathrm{pCO}_{2}>49 \mathrm{~mm}\right.$ $\mathrm{Hg}$; bicarbonate $>28 \mathrm{mmol} / \mathrm{l}$ ) and daytime somnolence, assessed using the Epworth sleepiness scale ${ }^{27}$ (ESS) where a score of 10 or above indicates abnormally excessive sleepiness. A control group of 10 patients with ALS without any evidence of respiratory difficulty or sleep disturbance was also recruited, using the above criteria, who were broadly matched to the experimental group for age, sex, and disease severity, measured on the ALS functional rating scale. ${ }^{28}$ The NIPPV group consisted of nine men, and the control group consisted of seven men and three women. Other clinical data are given in table 1. All participants were recruited from the Motor Neurone Disease Care and Research Centre at King's College Hospital, London and all investigations were undertaken at the Department of Respiratory Medicine, King's College Hospital. The study was approved by the Maudsley Hospital ethics committee (research) and the King's College Hospital ethics committee.

PROCEDURE

Informed consent was obtained from each patient before starting the study. For the NIPPV group, neuropsychological assessment was carried out on the morning after polysomnography. They were then provided with a portable ventilator, fitted with a mask, and fully instructed in its use during an inpatient stay. After successful initiation of ventilation, the patients returned home where ventilation was used every night for about 6 weeks. If any problems arose during this time, they were able to return to the clinic for advice and for any necessary adjustments to the ventilator or mask to be made. After about 6 weeks of ventilation, each patient returned to the clinic where repeat daytime blood gas measurements were recorded and the neuropsychological assessment was repeated.

For the control group, an initial interview was conducted with the respiratory physician at the clinic, daytime blood gas tensions and forced vital capacity were recorded, and the ESS was administered, to ensure that each patient was not experiencing any respiratory difficulties or hypoventilation. The neuropsychological assessment was then carried out and the patients returned home. Most of these patients returned to clinic about 6 weeks later, when the interview, vital capacity, ear lobe blood gas, ESS, and neuropsychological tests were repeated. As, for practical reasons, some control patients were unable to return to the department at the 6 week stage, the second neuropsychological assessment was conducted at their home, and thus blood gas and vital capacity measurements were not repeated at this stage in all of this group. However, at the time of the second interview, a clinical assessment was made and the ESS administered to ensure that no symptoms of hypoventilation or sleep disturbance had developed. Blood gas and vital capacity measurements were made subsequently when the patient next attended the hospital, confirming the absence of hypoventilation.

Regardless of location, the second assessment was carried out at the same time of day as the first (9 30-11 $00 \mathrm{am}$ ), and patients were seated upright for assessments.

NEUROPSYCHOLOGICAL ASSESSMENT

Six standardised tests of memory, attention, and executive function were administered to assess a range of cognitive functions. These were selected for their relative invulnerability to the effects of possible motor or speech disturbances in ALS, and where possible tests with more than one version were chosen so that valid testing could be repeated 6 weeks later. In all cases where two versions of the same test were used, half the participants received one version first, and half received the other version first. In addition, the test selection was short so as to minimise the stress and effort involved in the assessment.

Two versions of the forward digit span test were used, one from the Wechsler adult intelligence scale- revised $^{29}$ and the other from the Wechsler memory scale-revised. ${ }^{30}$ This task

Table 1 Comparison of characteristics of the NIPPV and control groups at assessment 1 (mean (SD))

\begin{tabular}{lccl}
\hline & NIPPV group $(n=9)$ & Control group $(n=10)$ & $p$ Value \\
\hline Age (y) & $61.11(7.83)$ & $61.90(6.97)$ & 0.720 \\
ALSFRS symptom severity & $26.00(2.00)$ & $27.67(5.43)$ & 0.428 \\
Interval between assessments (days) & $45.11(9.93)$ & $39.80(8.73)$ & 0.211 \\
Arterialised ear lobe blood CO $\mathrm{C}_{2}$ partial pressure $(\mathrm{mm} \mathrm{Hg})$ & $49.95(4.88)$ & $35.03(2.10)$ & $0.000^{\star \star \star}$ \\
Arterialised ear lobe blood bicarbonate (mmol/l) & $31.97(1.45)$ & $23.83(1.80)$ & $0.000^{\star \star \star}$ \\
ESS score & $9.14(5.27)$ & $4.22(2.11)$ & $0.012^{\star}$ \\
Forced vital capacity (l) & $2.01(0.51)$ & $3.67(0.86)$ & $0.001^{\star \star}$ \\
HAD Anxiety score & $2.80(3.03)$ & $4.22(3.49)$ & 0.681 \\
HAD Depression score & $1.33(2.31)$ & $1.89(1.05)$ & 0.518 \\
\hline
\end{tabular}

${ }^{\star} \mathrm{p}<0.05 ;{ }^{\star \star} \mathrm{p}<0.01 ;{ }^{\star \star \star} \mathrm{p}<0.001$. 
requires the subject to repeat strings of digits of increasing length, measuring short term auditory memory span. Raw scores were used for this test, with one point for every string correctly repeated.

A written version of the verbal fluency test, ${ }^{31}$ devised by Abrahams et $a l^{4233}$ was used as a measure of executive function. This is a commonly used test of intrinsic response generation in which the subject has 5 minutes to write down as many words as they can beginning with letter "S", followed by 4 minutes to write down as many four letter words as they can beginning with the letter " $C$ ". A motor control is then introduced by later timing the subject as they copy out all the words they have written down. A verbal fluency index (VFI) is calculated as follows:

Time allowed ( 9 minutes)-time taken to copy all words generated divided by

total number of words generated

The VFI therefore represents the average "thinking time" required for each word, so a low value indicates better performance. Only one version of this task has been systematically used with clinical populations, and it is not known what test-retest interval is required to eliminate the possibility of practice effects. Normative data for this test have produced a mean VFI of 4.9 (SD 2.7). ${ }^{32}$

For the Kendrick object learning test (KOLT), ${ }^{34}$ participants look at four cards showing line drawings of common objects. The four cards are presented consecutively, each one with more pictures than the last, and presentation time is longer each time, allowing 3 seconds for each picture on the card. After each presentation, the card is removed and the subject must recall as many of the objects as they can. The total number is calculated from all four cards at the end, and can then be converted into an age related standardised score using normative tables. However, because the age range of our participants was greater than that used in the standardisation studies for the KOLT, and because our two groups were well matched for age, the raw score was considered a valid performance index and was therefore used. This test primarily measures short term visual memory and learning, but also involves use of strategy and semantic clustering.

Two subtests from the adult memory and information processing battery ${ }^{35}$ were also administered. As a consequence of the variability in patients' motor function the graphic tasks (figure recall and design learning) were omitted. The story recall task provides a measure of immediate and delayed verbal memory, by requiring the participant to recall as much as they can from a short story, both immediately after it is read to them, and 30 minutes later. The list learning task, in which a list of 15 words must be acquired over a maximum of five trials (list learning) and then retained after a distractor trial (list recall), was used to assess incremental verbal learning and forgetting. The scoring for the two versions of these tests are not directly comparable because normative data differ slightly, so a standardised score was calculated from each raw score, using means (SD) obtained from the normative data tables (with a resulting average $=100($ SD 15)).

In addition, a measure of anxiety and depression-the hospital anxiety and depression scale $^{36}$ - was included to rule out the possibility that changes in mood were affecting the participants' cognitive function. As in previous studies $^{32}$ one question from this scale-"I feel slowed down"-was omitted, as it was thought that the patients' physical disability might exaggerate scores for this item. Raw scores were used from this questionnaire.

\section{STATISTICAL ANALYSIS}

The Mann-Whitney test was used to compare test results between groups, and the Wilcoxon test was used for within group comparisons over time.

\section{Results}

CLINICAL ASSESSMENT

The NIPPV and control groups were of similar age and general symptom severity, and the interval between the two assessments was comparable for both groups (table 1). As expected, the average forced vital capacity for the NIPPV group at the beginning of the study was significantly lower than that for the control group $(p=0.001)$. Blood concentrations of bicarbonate and carbon dioxide in the NIPPV group were abnormal at the first assessment. However, by the second assessment, levels of carbon dioxide and bicarbonate had significantly declined $(\mathrm{p}=0.001)$ to within normal limits, indicating normal ventilation (table 2). In addition, clinical symptoms of hypoventilation, including daytime sleepiness as measured by the ESS disappeared. By contrast, blood gas measurements for the control group were normal at the first and second (in those tested) assessment, and there were no symptoms of hypoventilation at either the first or second assessment.

Table 2 Clinical features of the NIPPV group $(n=9)$ at assessments 1 and 2

\begin{tabular}{lccc}
\hline & Assessment 1 Mean (SD) & Assessment 2 Mean (SD) & p Value \\
\hline $\begin{array}{l}\text { Arterialised ear lobe blood } \mathrm{CO}_{2} \\
\quad \text { partial pressure (mm Hg) }\end{array}$ & $49.95(4.88)$ & $42.45(3.83)$ & $0.008^{\star \star}$ \\
Arterialised ear lobe blood & & & $0.008^{\star \star}$ \\
$\quad$ bicarbonate (mmol/1) & $31.97(1.45)$ & $27.07(1.67)$ & $0.018^{\star}$ \\
ESS score & $9.14(5.27)$ & $4.57(3.82)$ & 0.581 \\
HAD Anxiety score & $2.80(3.03)$ & $3.40(1.82)$ & 0.317 \\
HAD Depression score & $1.33(2.31)$ & $1.67(2.89)$ & \\
\hline
\end{tabular}

${ }^{\star} \mathrm{p}<0.05 ;{ }^{\star \star} \mathrm{p}<0.01$. 
Table 3 Cognitive assessment results for the NIPPV group at assessments 1 and 2

\begin{tabular}{lccc}
\hline & $\begin{array}{c}\text { Assessment 1 } \\
\text { Mean (SD) }\end{array}$ & $\begin{array}{c}\text { Assessment 2 Mean } \\
(\text { SD) }\end{array}$ & p Value \\
\hline Digit span (raw score) & $8.63(2.56)$ & $9.25(2.19)$ & 0.301 \\
Story recall immediate (standardised score) & $92.80(11.62)$ & $99.36(13.50)$ & 0.176 \\
Story recall delayed (standardised score) & $93.53(11.98)$ & $97.40(9.72)$ & 0.237 \\
List learning (standardised score) & $85.58(9.07)$ & $100.13(8.88)$ & $0.017^{\star}$ \\
List recall (standardised score) & $90.29(11.44)$ & $99.24(11.86)$ & 0.093 \\
KOLT (raw score) & $37.14(5.84)$ & $43.00(3.37)$ & $0.040^{\star}$ \\
Verbal fluency index (seconds/word) & $10.99(8.11)$ & $8.45(5.65)$ & 0.063 \\
\hline
\end{tabular}

${ }^{\star} \mathrm{p}<0.05$.

COGNITIVE PERFORMANCE OF THE NIPPV GROUP

AT ASSESSMENTS 1 AND 2

The NIPPV group showed significant $(p<0.05)$ improvement on some cognitive tests after ventilation. For list learning over trials, the mean standardised score increased from 85.58 to $100.13(\mathrm{p}=0.017)$, whereas the KOLT raw score showed a smaller but significant improvement from 37.14 to $43.00(\mathrm{p}=0.040)$. The mean verbal fluency index (VFI) decreased from 10.99 to 8.45 , showing a trend towards improvement $(p=0.063)$ (a lower score on this test reflects a better performance). Mean scores for the other tests (list recall, story recall, digit span) also increased between the first and second assessment, but these changes did not reach significance. These data are shown in table 3 .

COGNITIVE PERFORMANCE OF THE CONTROL

GROUP AT ASSESSMENTS 1 AND 2

Significant changes were found for two tests. The KOLT raw scores decreased between the first and second assessments, suggesting a slight deterioration in performance. An improvement was found for immediate story recall in the control group $(p=0.050)$. Although the mean score on this test also increased in the NIPPV group, the change was not significant. As the stories used at each assessment time were different, it seems unlikely that practice effects could explain this difference. One possible interpretation of this result is that some of the control group participants underwent their second assessment at home, because of the difficulty of travelling to the hospital, whereas all of the NIPPV group participants were reassessed in the clinic. The more relaxed atmosphere in the home environment may have specifically facilitated the attentional capacity on which this task depends. The story recall test requires a single episode of sustained auditory attention to memorise a chain of complex information, rather than the incremental learning of smaller pieces of information required by the other memory tests. This could explain why story

Table 4 Cognitive assessment results for the control group at assessments 1 and 2

\begin{tabular}{lccc}
\hline & $\begin{array}{c}\text { Assessment 1 } \\
\text { Mean (SD) }\end{array}$ & $\begin{array}{c}\text { Assessment 2 } \\
\text { Mean (SD) }\end{array}$ & p Value \\
\hline Digit span (raw score) & $8.78(1.20)$ & $9.11(1.76)$ & 0.546 \\
Story recall immediate (standardised score) & $103.51(13.27)$ & $114.54(16.53)$ & 0.050 \\
Story recall delayed (standardised score) & $104.31(16.70)$ & $111.19(14.89)$ & 0.176 \\
List learning (standardised score) & $108.70(7.66)$ & $108.11(16.09)$ & 0.799 \\
List recall (standardised score) & $110.34(11.39)$ & $101.15(19.68)$ & 0.169 \\
KOLT (raw score) & $50.38(5.78)$ & $46.38(9.10)$ & $0.042^{\star}$ \\
Verbal fluency index (seconds/word) & $6.93(5.08)$ & $6.07(4.59)$ & 0.161 \\
\hline
\end{tabular}

${ }^{\star} \mathrm{p}<0.05$. recall performance might be selectively influenced by the test environment.

There was concern that practice effects might affect results for the verbal fluency task, as only one version of the test was used. This is unlikely as the change in the mean score for the control group on the second assessment occasion was negligible $(\mathrm{p}=0.463)$. Mean scores at assessments 1 and 2 for all tests are shown in table 4 .

COGNITIVE PERFORMANCE OF THE NIPPV GROUP COMPARED WITH THE CONTROL GROUP A comparison of average scores for the NIPPV group and control group at assessment 1 yielded highly significant results for list learning $(p=0.001)$, list recall $(p=0.006)$, verbal fluency $(p=0.001)$, and KOLT $(p=0.001)$, with the NIPPV group performing at a much lower level than the control group. However, these very marked differences disappeared after ventilation, list learning $(\mathrm{p}=0.142)$, list recall $(\mathrm{p}=0.417)$, verbal fluency $(\mathrm{p}=0.525)$, and KOLT $(p=0.457)$.

CHANGE IN COGNITIVE PERFORMANCE OF THE NIPPV GROUP COMPARED WITH THE CONTROL GROUP

Finally, the change in performance (score at assessment 2 -score at assessment 1 ) on all the cognitive tests was calculated for each group. When these were compared between the groups, there were significant differences for three measures: list learning $(p=0.013)$, list recall $(p=0.026)$, and KOLT $(p=0.007)$. In each case the NIPPV group showed a greater score increase than the control group.

MOOD

Levels of anxiety and depression were low for both groups, with all individual scores well below the cut off for clinical significance. In addition, mean scores did not differ significantly between the groups on either assessment occasion, and no significant changes were found between assessment times for either group (tables 1 and 2).

\section{Discussion}

In this study, patients with ALS with sleep disturbance and nocturnal hypoventilation have shown significant deficits on three tests of memory and one measure of executive function, when compared with a group of patients with ALS without respiratory symptoms. A significant improvement in performance on two of these measures of memory, and a trend towards improvement on the measure of executive function, has been shown after provision of NIPPV over a 6 week period to the patients with hypoventilation. Minimal change in cognitive function was seen in the control group after 6 weeks. These findings suggest that cognitive deficits may be caused by impaired nocturnal ventilation and sleep disturbance in ALS, and that these deficits could be improved by the administration of NIPPV in some cases. However, a large randomised controlled study will be necessary to test this 
hypothesis fully as the small numbers and nonblind nature of this trial weaken the current evidence somewhat.

Obstructive sleep apnoea syndrome (OSAS) may be responsible for a deterioration in cognitive function, ${ }^{16-18}$ some of which can be reversed with successful treatment of the sleep apnoea. ${ }^{1920}$ However, the relation between these phenomena is not fully understood. Both disturbed sleep architecture and nocturnal oxygen desaturation are thought to directly contribute to the excessive daytime sleepiness that characterises OSAS, ${ }^{37}$ and it has been shown that impaired vigilance is an important factor underlying poor cognitive performance. ${ }^{18}{ }^{39}$ Several studies have attempted to distinguish between these effects. A recent sleep apnoea treatment study ${ }^{40}$ demonstrated normalisation of most neuropsychological deficits after treatment, with the exception of verbal fluency, planning abilities, and manual dexterity. With reference to previous results showing that such deficits could be largely attributed to severity of nocturnal hypoxaemia in OSAS, ${ }^{18}$ the authors concluded that irreversible anoxic brain damage was responsible for these permanent impairments. This is consistent with another study ${ }^{41}$ which suggests that vigilance impairment may account for deficits on some tests, which were found to be reversible with ventilation, but that in a subgroup of hypoxaemic patients other areas of cognitive function do not improve after treatment.

The current findings indicate that changes in memory and some improvement in executive function (verbal fluency) could result from treatment of hypoventilation in ALS. This pattern is similar to that described in the OSAS literature, although verbal fluency has not been found to improve post-treatment in studies of sleep apnoea. Analysis of the data shows that although a fairly substantial improvement in the mean VFI occurred for the NIPPV group, the mean scores at the second assessment were still considerably poorer than those for the normative sample. ${ }^{32}$ This could be interpreted as evidence for a combination of two or three factors affecting verbal fluency performance: a reversible attention/vigilance impairment, in addition to permanent hypoxaemic damage and/or permanent abnormalities in the dorsolateral prefrontal cortex (previously shown to affect verbal fluency performance in a proportion of patients with $\left.\mathrm{ALS}^{4}{ }^{33}\right)$. Future studies of hypoventilation in ALS could examine this question more thoroughly. It would also be useful to incorporate some of the other cognitive tests used in the OSAS studies to allow a more direct comparison of the cognitive profiles associated with the two syndromes. Inclusion of patient and carer reported symptom questionnaires in future studies could also provide an interesting dimension.

The results of this study have important implications for the interpretation of investigations of cognitive function in ALS. It is unlikely that nocturnal hypoventilation can entirely account for the cognitive deficits found in nondemented patients with ALS, because many patients evaluated in previous studies were not thought to have significant respiratory difficulty. However, respiratory difficulties may exacerbate cognitive impairment in ALS. It is possible that hypoventilation has contributed to previous findings of mild memory and executive impairments in ALS, and this may in part explain why estimates of the prevalence of these deficits have fluctuated so widely across studies. Future studies of cognitive function in ALS should control for sleep disturbance and hypoventilation. In addition, the current findings suggest that cognitive impairment may represent one factor contributing to the decline in quality of life experienced by patients with ALS with hypoventilation. Further investigation is needed to determine the extent to which hypoventilation contributes to cognitive difficulties in non-demented patients with ALS. Normative test data for all measures could not validly be used for this purpose in this study, as the age range of the participants fell slightly outside that of the standardisation sample, but age matching between groups was used to overcome this difficulty.

The design of this study could be improved by the inclusion of measures of intelligence in the test battery. Without them, it is not possible to be absolutely certain that the discrepancies found in cognitive performance between the two groups at the first assessment are a pure reflection of the differing ventilatory function of the two groups. However, as these differences disappeared after ventilation, it is likely that this was the major factor responsible.

In conclusion, these findings raise the possibility that hypoventilation impairs cognitive function in ALS, and that these impairments could be reduced by the provision of nocturnal ventilation. A randomised controlled trial to resolve this question would be of great benefit.

ICN-D is supported by the Medical Research Council, UK. Support has also come from the Motor Neurone Disease Association, through the facilities of the King's MND Care and Research Centre, and from the Psychiatry Research Trust, trophy Association of . RAL is

1 Kew JJM, Leigh PN, Playford ED, et al. Cortical functions in amyotrophic lateral sclerosis. Brain 1993;116:655-80.

2 Kew JJM, Goldstein LH, Leigh PN, et al. The relationship between abnormalities of cognitive function and cerebral activation in amyotrophic lateral sclerosis. Brain 1993;116: 1399-423.

3 Talbot PR, Goulding PJ, Lloyd JJ, et al. Inter-relation between "classic" motor neuron disease and frontotemporal dementia: neuropsychological and single photon emission computed tomography study. I Neurol Neurosurg Psychiatry 1995;58:541-7.

4 Abrahams S, Goldstein LH, Kew JJM, et al. Frontal lobe dysfunction in amyotrophic lateral sclerosis. Brain 1996; 119:2105-20.

5 Gallassi R, Montagna P, Ciardulli C, et al. Cognitive impairments in motor neurone disease. Acta Neurol Scand ments in moto

6 Gallassi R, Montagna P, Morreale A, et al. Neuropsychologi$\mathrm{cal}$, electorencephalogram and brain computed tomography findings in motor neurone disease. European Neurology 1989;29:115-20.

7 David AS, Gillham RA. Neuropsychological study of motor neurone disease. Psychosomatics 1986;27:441-5

8 Ludolph AC, Langen KJ, Regard M, et al. Frontal lobe function in amyotrophic lateral sclerosis: a neuropsychoogic and positron emission tomography study. Acta Neurol Scand 1992;85:81-9.

9 Kato S, Hayashi H, Yagashita A. Involvement of the frontotemporal lobe and limbic system in amyotrophic lateral sclerosis: as assessed by serial computed tomophic lateral sclerosis: as assessed by serial computed tomography and

10 Kimura K, Tachibana N, Kimura J, et al. Sleep-disordered breathing at an early stage of amyotrophic lateral sclerosis. f Neurol Sci 1999;164:37-43. 
11 Ferguson KA, Strong MJ, Ahmad D, et al. Sleep-disordered breathing in amyotrophic lateral sclerosis. Chest 1996;110 breathin

12 Gay PC, Westbrook PR, Daube JR, et al. Effects of alterations in pulmonary function and sleep variables on survival in patients with amyotrophic lateral sclerosis. Mayo Clin Proc 1991;66.

13 Kaplan LM, Hollander D. Respiratory dysfunction in amyotrophic lateral sclerosis. Clin Chest Med 1994;15:67581.

14 Hetta J, Jansson I. Sleep in patients with amyotrophic lateral sclerosis. F Neurol 1997;244:S7-9.

15 Aboussouan LS, Lewis RA. Sleep, respiration and ALS. $\mathcal{F}$ Neurol Sci 1999;164:1-2.

16 Naeegele B, Thouvard V, Pepin JL, et al. Deficits of cognitive executive functions in patients with sleep apnoea syndrome. Sleep 1995;18:43-52

17 Kelly DA, Claypoole KH, Coppel DB. Sleep apnoea syndrome: symptomatology, associated features, and neuryndrome: symptomatology, associated features, and neu-

18 Bedard MA, Montplaisir J, Richer F, et al. Obstructive sleep apnoea syndrome: pathogenesis of neuropsychological deficit. F Clin Exp Neuropsychol 1991;13:950-64.

19 Scheltens P, Visscher F, Van Keimpema AR, et al. Sleep apnoea syndrome presenting with cognitive impairment. Neurology 1991;4:155-6.

20 Feuerstein C, Naeegele B, Pepin JL, et al. Frontal lobe-related cognitive functions in patients with sleep apnoea syndrome before and after treatment. Acta Neurol Belg 1997;97:96-107.

21 Sherman MS, Paz HL. Review of respiratory care of the patient with amyotrophic lateral sclerosis. Respiration 1994; 61:61-7.

22 Ellis ER, Bye PTP, Bruderer JW, et al. Treatment of respiratory failure during sleep in patients with neuromuscular disease: positive-pressure ventilation through a nose mask. Am Rev Respir Dis 1987;135:148-52.

23 Serisier DE, Mastaglia FL, Gibson GJ. Respiratory muscle function and ventilatory control. I. In patients with motor function and ventilatory control. I. In patients with motor neurone disease. II In patie

24 Bach JR. Amyotrophic lateral sclerosis: predictors for prolongation of life by non-invasive respiratory aids. Arch Phys Med Rehabil 1995;76:823-32.

25 Pinto AC, Evangalista T, Carvalho M, et al. Respiratory assistance with a non-invasive ventilator (BIPAP) in MND/ ALS patients: survival rates in a controlled trial. $\mathcal{f}$ Neurol Sci 1995;129:S19-26.
26 Swash M, Leigh PN. Criteria for diagnosis of familial amyotrophic lateral sclerosis. Neuromusc Disord 1992;2:7-9.

27 Johns MW. A new method of measuring daytime sleepiness: the Epworth sleepiness scale. Sleep 1991;14:540-5.

28 The ALS CNTF treatment study (ACTS) phase I-II study group. The amyotrophic lateral sclerosis functional rating scale. Arch Neurol 1996;53:141-7.

29 Wechsler D. WAIS-R manual. Cleveland, OH: The Psychological Corporation, 1981.

30 Wechsler D. WMS-R manual. Cleveland, OH: The Psychological Corporation, 1987.

31 Thurstone LL, Thurstone TG. Primary mental abilities. Chicago: Science Research Associates, 1962.

32 Abrahams S, Goldstein LH, Al-Chalabi A, et al. Relation between cognitive dysfunction and pseudobulbar palsy in amyotrophic lateral sclerosis. F Neurol Neurosurg Psychiatry 1997;62:464-72.

33 Abrahams S, Leigh PN, Harvey A, et al. Verbal fluency and executive dysfunction in amyotrophic lateral sclerosis. Neuropsychologia 2000;38:734-47.

34 Kendrick DC. Kendrick cognitive tests for the elderly. Windsor: NFER-Nelson, 1985.

35 Coughlan AK, Hollows SE. The adult memory and information processing battery. Leeds: AK Coughlan, 1985.

36 Zigmond AS, Snaith RP. The hospital anxiety and depression scale. Acta Psychiatr Scand 1983;67:361-70.

37 Bedard MA, Montplaisir J, Malo J, et al. Sleep disruption and nocturnal hypoxemia as determinants of vigilance impairment in sleep apnoea syndrome. Chest 1991;100: impairm

38 Guilleminault C, Partinen M, Quera-Salva MA, et al. Determinants of daytime sleepiness in obstructive sleep apnoea. Chest 1988;94:32-7.

39 Cheshire K, Engleman H, Deary I, et al. Factors impairing daytime performance in patients with sleep apnoea/ hypopnoea syndrome. Arch Intern Med 1992;152:538-41.

40 Bedard MA, Montplaisir J, Malo J, et al. Persistent neuropsychological deficits and vigilance impairment in sleep apnoea syndrome after treatment with continuous positive airway pressure (CPAP). F Clin Exp Neuropsychol 1993;15:330-41.

41 Valencia-Flores M, Bliwise DL, Guilleminault C, et al. Cognitive function in patients with sleep apnoea after acute nocturnal nasal continuous positive airway pressure
(CPAP) treatment: sleepiness and hypoxemia effects. $\mathcal{F}$ Clin Exp Neuropsychol 1996;18:197-210. 